\title{
TRADUCCIÓN: EL PROYECTO RITMANALÍTICO ${ }^{1}$
}

\section{TRANSLATION: THE RHYTHMANALITIC PROJECT}

\author{
Henri Lefebvre \\ Catherine Régulier \\ Traductor: Roy Alfaro Vargas*
}

TEORÍA SOCIAL

RESUMEN

Este ensayo presenta el concepto de ritmanálisis como parte del estudio de la vida cotidiana. Tal concepto se establece no solo en términos sociológicos, sino también en términos físico-biológicos. Así, la noción de ritmo permite aprehender la interacción de los diferentes ámbitos que componen el mundo natural y psico-social, en relación con la economía capitalista. De hecho, el concepto de ritmo es aquí asumido como un fenómeno total, lo cual implica una investigación interdisciplinaria. Finalmente, con esta traducción, se intenta llenar el hueco existente, alrededor de esta noción, en las ciencias sociales desarrolladas en Latinoamérica.

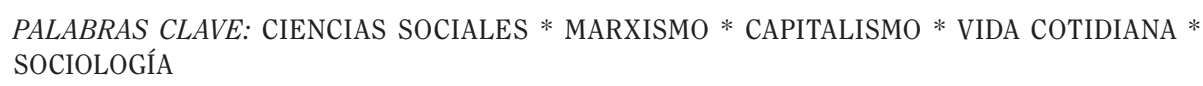

\section{ABSTRACT}

This essay puts forward the concept of rhythmanalysis as a part of the study of everyday life. Such a concept is set up not only in sociological terms, but in physico-biological terms as well. Thus, the notion of rhythm permits apprehending the interaction of the different realms that are components of our natural and psycho-social world vis-à-vis the capitalist economy. As a matter of fact, the concept of rhythm is here assumed as a total phenomenon, which implies an interdisciplinary research. Finally, with this translation, we intend to fill the existing gap, around this notion, in the social sciences developed in Latin America.

KEYWORDS: SOCIAL SCIENCES * MARXISM * CAPITALISM * EVERYDAY LIFE * SOCIOLOGÍA

1 La referencia original del texto en francés es: Lefebvre Henri, Régulier Catherine, «Le projet rythmanalytique», En: Communications, 41, 1985. «L'espace perdu et le temps retrouvé», bajo la dirección de Rémy Lestienne et Edgar Morin. pp. 191-199.

DOI: https://doi.org/10.3406/comm.1985.1616

Investigador independiente, Costa Rica.

royalfarov@gmail.com

ID: 0000-0001-5893-2415 


\section{LA VIDA COTIDIANA Y LOS RITMOS}

En una obra por aparecer, nosotros mostraremos las relaciones entre la vida cotidiana y los ritmos, es decir, las modalidades concretas del tiempo social. El estudio ritmológico, que vamos a intentar, se integra al de la vida cotidiana. Este ahonda en ciertos aspectos de ella. La vida cotidiana se regula sobre el tiempo abstracto, cuantitativo, aquel de los relojes. Este tiempo se ha introducido poco a poco en Occidente, después de la invención de los relojes, en el curso de su entrada en la práctica social. Este tiempo homogéneo y desacralizado obtiene la victoria desde que él ha proporcionado la medida del tiempo de trabajo. A partir de ese momento histórico, él deviene el tiempo de la cotidianidad, subordinando a la organización del espacio los otros aspectos de lo cotidiano: las horas de dormir y de despertar, las horas de comida $y$ de la vida privada, las relaciones de los adultos con los niños, las distracciones $y$ el ocio, las relaciones en el lugar de habitación. Sin embargo, la vida cotidiana permanece recorrida $y$ atravesada por los grandes ritmos cósmicos y vitales: los días y las noches, los meses y las estaciones $y$, más precisamente aún, por los ritmos biológicos. De ello resulta en lo cotidiano una interacción perpetua entre estos ritmos y los procesos repetitivos ligados al tiempo homogéneo.

Esta integración tiene ciertos aspectos que dejaremos de lado, por ejemplo, los lazos tradicionales del tiempo social con las creencias $y$ las prescripciones religiosas. Nos consagraremos solamente al aspecto rítmico del tiempo cotidiano. El estudio de la vida cotidiana ya ha puesto en evidencia esta diferencia banal $y$, por tanto, ignorada, entre lo cíclico y lo lineal, entre el tiempo ritmado y el tiempo de las repeticiones brutales. Esta repetición es cansante, agotadora, fastidiosa, aunque el retorno del ciclo tiene el aspecto de un advenimiento $y$ de un evento. Su inicio, que, por ende, es solo un reinicio, tiene siempre la frescura de un descubrimiento $y$ de una invención. El inicio tiene siempre un encanto milagroso, el deseo, las ganas se renuevan milagrosamente.... Lo cotidiano es a la vez el lugar, el teatro y la puesta en juego de un conflicto entre los grandes ritmos indestructibles $y$ los procesos impuestos por la organización socioeconómica de la producción, del consumo, de la circulación y del hábitat. El análisis de la vida cotidiana muestra cómo y por qué el tiempo social es él mismo un producto social. Como todo producto, como el espacio, el tiempo se divide y se escinde en uso $y$ valor de uso, de un lado, $y$ en cambio $y$ valor de cambio del otro lado. De un lado se vende y del otro se vive.

De aquí una serie de hipótesis, que sirve de punto de partida para el ritmanálisis.

Primeramente, el tiempo cotidiano es doblemente medido o, más bien, él es a la vez mediente ${ }^{2}$ y medido. Por una parte, los ritmos fundamentales $y$ cíclicos se mantienen $y$, por otra parte, el tiempo cuantificado de los relojes impone algunas repeticiones monótonas. Los ciclos animan la repetición atravesándola. ¿No sería en razón de esta doble medida que la cotidianidad ha podido establecerse en los tiempos modernos, estabilizarse y, por así decir, institucionalizarse?

En segundo lugar, hay una lucha áspera y oscura alrededor del tiempo $y$ del empleo del tiempo. Esta lucha tiene los más sorprendentes prolongamientos. Los ritmos dichos naturales se modifican por razones múltiples, tecnológicas o socio-económicas, de una manera que exige investigaciones profundas.

En tercer lugar, el tiempo cuantificado se somete a una ley muy general de esta sociedad: él deviene a la vez uniforme y monótono, disgregándose y fragmentándose. Como el espacio, él se divide en lotes y en parcelas: transportes - ellos mismos fragmentados - trabajos diversos, distracciones, ocio. No hay tiempo para hacer todo, sino que cada "hacer" tiene su tiempo. Estos fragmentos se jerarquizan, pero el trabajo permanece en una larga medida (a pesar de una depreciación que combate una revalorización práctica en periodo de desempleo), lo esencial, el referente al cual se ensaya relacionar todo. No obstante, las perturbaciones rítmicas se multiplican, así como los problemas llamados nerviosos. No es inexacto decir que

$2 \quad$ "Mediente", el participio presente del verbo medir [Nota del traductor]. 
los nervios y el cerebro tienen sus ritmos, del mismo modo que el sentido y el intelecto.

En la perspectiva ritmanalítica, se pueden describir las "jornadas" y los empleos del tiempo según las categorías sociales, los sexos, las edades. De notar es que los objetos son consumidores del tiempo, se inscriben en su empleo con sus exigencias propias. Una máquina de lavar consume su fragmento de tiempo (funcionamiento y mantenimiento) como ocupa su fragmento de espacio. Las horas de comida resultan de convenciones sociales, ya que estas difieren según el país. Pero, si usted come a medio día y a las ocho horas de la tarde, usted termina por tener hambre a estas horas. Es preciso quizás dos decenas de años para acostumbrar al cuerpo a estos ritmos y no es raro que los niños rechacen los ritmos sociales. En cuanto a la concentración intelectual $y$ a las actividades que ella implica (lectura, escritura, análisis), estas tienen su ritmo propio, creado por el hábito, o sea, por un compromiso más o menos armonioso entre lo repetitivo, lo cíclico y esto que sobreviene. Estos comportamientos adquiridos según una cierta partición del tiempo y según algunos ritmos bien definidos dejan, por tanto, la impresión de espontaneidad. ¿Automatismos o espontaneidad? Se atribuye a una necesidad esencial esto que resulta de obligaciones externas. Aquel que se despierta a las seis de la mañana porque está así ritmado por su trabajo, tiene quizás sueño y necesidad de dormir. ¿Esta interacción de lo repetitivo y de lo rítmico no engendra, más o menos rápidamente, una desposesión del cuerpo? Esta desposesión ha sido ya notada $y$ señalada algunas veces, sin que uno haya asido todas las razones de ello.

Lo relativo, en la vida cotidiana, en las relaciones sociales, aparece así en cada "sujeto" como necesario y absoluto, como esencial $y$ auténtico. Aunque se introduzca un elemento nuevo en el tiempo cotidiano, esta construcción vacila y amenaza con derrumbarse, mostrando por allá que ella no era necesaria, ni auténtica. Devenir insomne, amoroso o bulímico, provoca entrar en otra cotidianidad...

La organización ritmada del tiempo cotidiano es, en un sentido, esto que hay de más personal, interior. Y es así lo que hay de más exterior (esto que corresponde a una fórmula célebre de Hegel). No se trata de una ilusión ni de una ideología, sino de una realidad. Los ritmos adquiridos son a la vez interiores $y$ sociales. En una jornada del mundo moderno, todo el mundo hace aproximadamente la misma cosa aproximadamente a las mismas horas, pero cada uno es realmente único en hacerlo.

Lo cíclico y lo lineal son categorías, es decir, nociones o conceptos. Cada una de estas dos palabras designa - denota - una extrema diversidad de hechos y de fenómenos. Los movimientos y los procesos cíclicos —ondulaciones, vibraciones, retornos y rotacionesson innumerables, desde lo microscópico hasta lo astronómico, desde las moléculas a las galaxias, pasando por las pulsaciones del corazón o el movimiento de los párpados, la respiración, la alternancia de los días y las noches, de los meses y de las estaciones, etc. En cuanto a lo lineal, señala sin importar cuál serie de hechos idénticos, separados por un intervalo de tiempo más o menos grande: la caída de una gota de agua, el golpe de un martillo, el ruido de un motor, etc. La connotación no desaparece en la denotación de estos términos. Lo cíclico tiene para él una apreciación más bien favorable: proviene del cosmos, de lo global (mondial), de la naturaleza. Cada uno puede representarse en las olas del mar — buena representación, plena de sentido - o en las ondas sonoras, o bien en los ciclos circadianos o mensuales. Lo lineal, él mismo, no se representa como monótono, agotante y asimismo intolerable.

Las relaciones de lo cíclico y de lo lineal -interacciones, interferencias, dominación del uno sobre el otro o rebelión del uno contra el otro- no son simples: hay entre ellos una unidad conflictual. Ellos se penetran, pero en una lucha incesante, a veces compromiso, a veces perturbación. Sin embargo, hay entre ellos una unidad indisoluble: en el reloj, lo repetitivo, el tic-tac, mide el ciclo de las horas y de los días, y recíprocamente. En la práctica industrial, donde lo repetitivo lineal tiende a arrebatarlo, la lucha es intensa.

Si pues lo cíclico y lo lineal son categorías del tiempo y del ritmo con características 
generales (pues la medida, aquella de lo uno por lo otro, lo que hace de cada uno de ellos un mediente-medido), ¿no hay otras categorías? ¿Otros rasgos característicos del tiempo y del ritmo? ¿Otro tiempo?

El tiempo que provisoriamente denominaremos "apropiado" tiene sus características propias. Normal o excepcional, es un tiempo que olvida el tiempo, después del cual el tiempo no (se) cuenta más. Él adviene o sobreviene cuando una actividad aporta una plenitud, la que esta actividad sea banal (una ocupación, un trabajo) o sutil (mediación, contemplación), espontánea (juego del niño y también de los adultos) o sofisticada. Esta actividad se entiende a sí misma y al mundo. Ella tiene algunos rasgos de una autocreación y de un don más bien que una obligación o una imposición venida desde afuera. Ella es en el tiempo: ella es un tiempo, pero no lo refleja.

Para plantear bien la cuestión de los ritmos, volvamos a la vida cotidiana y a la descripción de una jornada. El empleo del tiempo la fragmenta, la divide en parcelas. Un cierto realismo consiste en una descripción minuciosa de estas parcelas: él estudia las actividades relativas a la alimentación, al vestido, a la limpieza, a los transportes, etc. Él menciona los productos empleados. Una tal descripción parecerá científica: ahora bien, ella pasa al lado del objeto mismo que no es la serie de los lapsos de tiempo así pasados, sino su encadenamiento en el tiempo, pues su ritmo. Lo esencial se perderá en provecho de lo accidental mismo y sobre todo si el estudio de los fragmentos permite teorizar algunas estructuras de lo cotidiano.

\section{2. ¿QUÉ ES EL RITMO?}

Cada uno cree saber lo que significa esta palabra. En efecto, cada uno lo siente de una manera empírica muy alejada de un conocimiento: el ritmo entra en lo vivido; ahora bien, esto no quiere decir que entre en lo conocido. De una constatación a una definición hay distancia $y$, más aún, del asir un ritmo cualquiera (aquel de un aire musical o de la respiración o de los latidos del corazón) a la concepción que aprehenda la simultaneidad y la complicación de varios ritmos, su unidad en su diversidad. Y, por tanto, cada uno de nosotros es esta unidad de relaciones diversas en las cuales los aspectos se subordinan a la acción hacia el exterior, orientándose hacia el afuera, hacia el Otro y el Mundo, a tal punto que se nos escapan. Solo tenemos consciencia de la mayor parte de nuestros ritmos, cuando comenzamos a sufrir un desregulamiento. Es en la unidad orgánica, psicológica y social de lo "percibiente", orientado hacia lo percibido, o sea, hacia los objetos, hacia los alrededores y hacia las otras personas, que se dan los ritmos que componen esta unidad. Se trata aquí del deseo y de las ganas, del sueño y de la vigilia, del sexo y de la actividad intelectual, etc.

Para que haya ritmo, es preciso que haya repetición en un movimiento, pero no importa cuál repetición. El retorno monótono del mismo idéntico ruido en sí no forma más un ritmo que un objeto móvil cualquiera que sea su trayectoria, por ejemplo, una piedra que cae. Aunque nuestra oreja $y$, sin duda, nuestro cerebro tienden a introducir un ritmo en toda repetición, mismo completamente lineal. Para que haya ritmo, es preciso que aparezcan en el movimiento unos tiempos fuertes $y$ otros débiles, que revengan según una regla o ley —unos tiempos largos y unos breves, retomados de manera reconocible - algunas paradas, algunos silencios, algunos espacios, algunas retomas y algunos intervalos según una regularidad. El ritmo comporta pues un tiempo diferenciado, una duración cualificada. Así pues, repeticiones, rupturas y retomas en este tiempo. Pues una medida, mas una medida interna, que se distinga fuertemente sin por tanto separarse la medida externa de ella, el tiempo $t$ (tiempo del reloj o del cronómetro) solo consistente en un parámetro homogéneo y cuantitativo. La medida externa puede $y$ debe superponerse a la medida interna en una reciprocidad de la acción, pero no pueden confundirse. No tienen ni el mismo comienzo, ni el mismo fin o finalidad. Esta doble medida entre la definición del ritmo, de su cualidad, irreducible a una determinación simple, implicando, al contrario, algunas relaciones complejas (dialécticas). Solo así pues un movimiento no mecánico puede tener un ritmo: esto que clasifica todo lo que se 
levanta de la pura mecánica en el dominio de lo cuantitativo, liberado abstractamente de la cualidad. No obstante, es preciso formular algunas reservas sobre esta afirmación. Por ejemplo, hay relaciones estrechas entre los ritmos $y$ los movimientos ondulatorios que se estudian matemática y físicamente. Los sonidos, estos elementos del movimiento musical, resultan con sus propiedades y combinaciones (alturas, frecuencias de las vibraciones, lugar sobre la escala de sonidos, o sea, sobre el continuo que va de lo grave a lo agudo, intensidades y timbres) - los sonidos resultan de vibraciones complejas, de movimientos ondulatorios que entran en los acordes y las armonías-. Se volverá más adelante $y$ de manera más profunda sobre la relación entre la musicalidad y el ritmo. Por el instante, basta notar que el ritmo supone:

a) Algunos elementos temporales bien marcados, acentuados, pues contrastantes, incluso opuestos como los tiempos fuertes y débiles.

Un movimiento de conjunto que trae con él todos estos elementos (por ejemplo, un movimiento de vals, más o menos rápido). Por este doble aspecto, el ritmo entra en una construcción general del tiempo, del movimiento, del devenir. Y por consecuencia en su problemática filosófica: repetición y devenir, relación de lo Mismo y lo Otro. Es de notar, de aquí en adelante, que el ritmo comportando una medida, implica una cierta memoria. Aunque la repetición mecánica se ejecuta reproduciendo el instante que precede, el ritmo conserva y la medida inicia el proceso y el recomienzo de este proceso con sus modificaciones, así pues con su multiplicidad y pluralidad. Sin repetir idénticamente lo "mismo", pero subordinándolo a la alteridad, incluso a la alteración, o sea, a la diferencia.

Para asir de manera sensible, pre-conceptualmente pero viva, el ritmo y los poliritmos, basta ver atentamente la superficie del mar. Las olas se suceden: toman forma en la cercanía de la playa, del acantilado, de las orillas. Estas olas tienen un ritmo que depende de la estación, del agua y de los vientos, pero también del mar que las trae, que las aporta. Cada mar tiene su ritmo, el del Mediterráneo no es el de los océanos. Pero vea bien cada ola. Sin cesar cambia. Aproximándose a la ribera, recibe el choque de la resaca: lleva numerosas olitas y hasta unos ínfimos estremecimientos que ella orienta pero que no van siempre en su dirección. Las ondas y las ondulaciones se caracterizan por la frecuencia, por la amplitud, por la energía desplazada. Observando las olas, se constata fácilmente esto que los físicos llaman la superposición de los pequeños movimientos. Las fuertes olas se enfrentan con algunos chorros de espuma, se interfieren ruidosamente. Las pequeñas ondulaciones se atraviesan las unas a las otras, amortiguándose más bien que enfrentándose. Si hay una corriente o algunos objetos sólidos animados de un movimiento propio, usted podrá ver la intuición de esto que es un campo poli-rítmico $y$, asimismo, entrever las relaciones entre los procesos complejos y las trayectorias, entre los cuerpos y las ondulaciones, etc.

Ahora bien, hay una teoría general de los ritmos. Un largo hábito, ya señalado, separa el tiempo del espacio, a pesar de las teorías físicas contemporáneas, que plantean una relación entre ellos. Hasta un nuevo orden, estas teorías fracasan en dar una concepción unitaria que permitiría también comprender las diversidades (diferencias).

Y he aquí ahora la hipótesis ritmanalítica. ¿El cuerpo? ¿Su cuerpo? Él consiste en un paquete de ritmos. ¿Por qué no decir un arreglo floral? ¿O un ramo? Porque estos términos connotan un orden estético, como si la naturaleza - artista - hubiera preparado y acondicionado la belleza, la armonía de los cuerpos. Esto quizás no es erróneo, pero aquí viene prematuramente dado. El cuerpo viviente - polirrítmico- se compone de ritmos diversos, cada "parte", cada órgano o función, teniendo la suya, en una interacción perpetua, en equilibrio sin duda "meta-estable", siempre comprometido y más a menudo restablecido, salvo en caso de problemas. ¿Cómo? ¿Por un mecanismo simple? ¿Por una homeostasis al 
modo cibernético? ¿O de manera más sutil, por ejemplo, por una jerarquía de centros y por un centro superior ordenando la actividad racional? Es una de nuestras cuestiones. Pero los alrededores del cuerpo, lo social tanto como lo cósmico, son igualmente unos paquetes de ritmos ("paquetes" en el sentido donde uno dice, no peyorativamente, como un acorde complejo, reuniendo diversas notas y diversos timbres, es un "paquete sonoro"). Vean ahora alrededor de ustedes esta pradera, este jardín, estos árboles, estas casas. Ellos se dan, se ofrecen a los ojos en una simultaneidad. Ahora bien, esta simultaneidad solo es hasta cierto punto apariencia, superficie, espectáculo. Vamos a profundizar. No teman enturbiar esta superficie, de poner en movimiento su nitidez. Hecha como este viento que sacude estos árboles. Que su mirada se haga penetrante, que no se limite más a reflejar, a espejear. Que transgreda un poco sus límites. Usted percibirá enseguida que cada planta, cada árbol tiene su ritmo. Y de igual modo varios ritmos: hojas, flores, frutos o granos. Sobre este cerezo, las flores, en primavera, nacen con las hojas que sobrevivirán a los frutos y caerán, no todas a la vez, en otoño. Desde entonces, usted va a asir "sinfónicamente" o "poli-rítmicamente" cada ser o cada estado, cada cuerpo, viviente o no viviente. Los asirá en su tiempo-espacio, en su lugar y su devenir próximo: hasta las casas y los edificios, hasta los pueblos y los paisajes.

¿Embustera, la simultaneidad? ¿Abusivo el sincronismo? No y sí. No: la cuasi-supresión de las distancias en el tiempo y el espacio por los medios actuales de comunicación no es desde luego sin importancia. Solo es "ver" el interés suscitado en la televisión por las informaciones en directo. Usted asiste a los eventos, en el instante donde pasa. Usted ve las masacres, los cadáveres, usted contempla las explosiones. Los misiles, los cohetes partiendo bajo sus ojos, yendo rápidamente hacia los objetivos. ¡Usted está ahí! - Pero no está usted ahí-. Usted tiene la pequeña impresión de estar ahí. ¡Subjetividad! Usted está en su sillón delante de una pequeña pantalla, bien llamada, ya que ella oculta esto que muestra. La simultaneidad no disimula solamente los dramas —o lo trágico-.
Ella enmascara el tiempo, la diacronía. ¿La historia? ¿La génesis? No solamente. La diversidad de los lugares, de los ritmos, pues de los países $y$ de los pueblos. Error simétrico y engaño corolario de la simultaneidad facticia: la devolución perpetua a la historia. ¡Ya que se trata de los tiempos presentes!

De estas primeras percepciones, resulta que el cuerpo viviente puede $y$ debe considerarse como interacción de órganos situados en él, teniendo cada uno su ritmo, pero sometido a una globalidad espacio-temporal. Además, este cuerpo humano es la sede y el lugar de interacción entre lo biológico, lo fisiológico (naturaleza), lo social (dicho a menudo "cultural"), cada uno de estos niveles, cada una de estas dimensiones, teniendo su especificidad, pues su espacio-tiempo: su ritmo. De ahí que choques inevitables (estrés), problemas, perturbaciones, en este conjunto en el cual nada garantiza absolutamente la estabilidad.

De ahí la importancia de las escalas, proporciones y ritmos. Para concebir la realidad física y su relación con la realidad fisiológica y sensible del ser humano, la filosofía moderna ha propuesto dos esquemas: el kantiano o neokantiano, el empírico o positivista. Según el primero, los fenómenos - los flujos de sensaciones - se clasifican, se ordenan, se organizan según dos categorías a priori, es decir, interiores al sujeto y a la consciencia, comprendidos aquí el tiempo y el espacio. El en-sí (lo nouménico) escapa a las conquistas de este "sujeto". Según el empirismo y el positivismo, los hechos sensibles se ordenan ellos mismos en relaciones de simultaneidad, de implicación, de encadenamiento. "Si A implica B y B implica C, A implica C". No hay necesidad de otras categorías que las de lo lógico o de la lógica, que no son por otro lado categorías sino evidencias experimentales, transcritas en un lenguaje formal.

Ahora bien, el conocimiento, de Newton a Einstein y la física contemporánea, sigue otra vía, marcada igualmente por ciertas filosofías como la de Feuerbach. Es exacto que solo percibimos nuestra relación con las cosas de la naturaleza, como con los objetos producidos, en una palabra, con las realidades. De suerte que es preciso distinguir entre las apariencias —que tienen ellas 
mismas una realidad - $y$ esto que hay efectivamente en estas cosas. Por ejemplo, ellas parecen inertes (esta mesa de madera, este lápiz, etc.) y, sin embargo, se mueven, o solo sería esto por el movimiento de la Tierra, al contrario, ellas contienen estos movimientos, energías: cambian, etc. Vale igualmente en las relaciones sociales como en la realidad física: este objeto, inmóvil delante de mí, es el producto del trabajo: es toda la cadena de mercancías que se disimula en él, objeto material y social. Por consecuencia, es preciso ir más allá de los hechos, de los fenómenos, del flujo de las sensaciones inmediatas, pero no están determinados de manera interior y puramente a priori ni de este lado, ni más allá del fenómeno y del hecho sensible, como lo creía la tradición kantiana.

Nuestra escala determina nuestro lugar, nuestra posición en el espacio-tiempo del universo: esto que percibimos en él y que sirve de punto de partida tanto para la práctica, como para el conocimiento teórico. Lo micro, así como lo macro se nos escapan, aunque pudiéramos alcanzarlos progresivamente por el conocimiento y por su relación con lo conocido. Nuestros ritmos nos insertan en un mundo vasto e infinitamente complejo: él nos impone una experiencia y los elementos de esta experiencia. Consideremos por ejemplo la luz. No la percibimos como una ondulación portadora de corpúsculos, sino como una maravilla metamorfoseando las cosas, como iluminación de objetos, como un juego en la superficie de todo lo que existe. Este lado subjetivo no contiene de ello sino una objetividad que ha permitido alcanzar, en el curso de largos siglos de investigaciones y de cálculos, una realidad física bajo los fenómenos luminosos, sin por otro lado definir exhaustivamente esta realidad.

El espectro de movimientos ondulatorios (conectados a algunas trayectorias o bien, al contrario, sin relación con ellas) se extiende indefinidamente, quizás infinitamente, de lo macro a lo micro, de los movimientos corpusculares a aquellos de las metagalaxias. El pensamiento relativista obliga a rechazar toda referencia fija $y$ definitiva. Un referencial solo puede ser provisional, coyuntural; y se puede reprochar a Einstein el haber rechazado el absoluto del espacio $y$ del tiempo newtoniano $y$, por tanto, conservar un absoluto, una constante del universo, la velocidad de la luz.

En el inmenso espectro, nosotros solo asimos $y$ percibimos esto que corresponde a nuestros propios ritmos, a los ritmos de nuestros órganos. Comprendidas aquí dos zonas inciertas y variables según los individuos, uno más allá de nuestras percepciones normales, hacia lo micro, y otro más allá, hacia lo macro (ondas sonoras y ultrasonidos, infrarrojo y ultravioleta, etc.). Se pueden, por otra parte, concebir dos seres en los cuales el campo de percepción estaría más extendido. Se pueden sobre todo producir algunos aparatos que extiendan efectivamente este campo. No persiste por esto menos con sus límites, sus bordes, sus fronteras.

El hombre (la especie): su ser físico y fisiológico es desde luego la medida del mundo, según la antigua fórmula de Protágoras. No es solamente que nuestro conocimiento sea relativo a nuestra constitución, es que el mundo que se ofrece a nosotros (la naturaleza, la Tierra y esto que llamamos el cielo, el cuerpo y su inserción en las relaciones sociales, etc.) es relativo a esta constitución. No a unas categorías a priori, sino a nuestros sentidos $y$ a los instrumentos de los cuales disponemos. Más filosóficamente: otra escala determinaría otro mundo. ¿El mismo?, sin duda, pero diferentemente asido.

Sin saberlo (esto que no quiere decir "inconscientemente"), la especie humana extrae en el seno del universo los movimientos que corresponden a sus propios movimientos. La oreja, los ojos y la vista, las manos no tienen nada de aparatos pasivos y simplemente registradores. Lo fabricado, lo formado, lo producido se instauran en esta escala, en la cual es preciso así comprender que no tiene nada de accidental o de arbitrario. Es la de la Tierra, la de los accidentes sobre la superficie terrestre y la de los ciclos que se desarrollan aquí. Esto no quiere decir que la producción se limite a reproducir las cosas y los objetos dados naturalmente. Lo creado no entra en esta escala, sea porque la supere, sea porque la trasfigure.

(París, marzo 1983) 
